\title{
Investigation on optical behaviour of hippuric acid for optoelectronics applications
}

\author{
Suresh Sagadevan ${ }^{1}$, Priya Murugasen ${ }^{2}$ \\ ${ }^{1}$ Department of Physics, Sree Sastha Institute of Engineering and Technology, Chennai-600 123, India \\ ${ }^{2}$ Department of Physics, Saveetha Engineering College, Chennai, India
}

Email address:

sureshsagadevan@gmail.com (S. Sagadevan)

To cite this article:

Suresh Sagadevan, Priya Murugasen. Investigation on Optical Behaviour of Hippuric Acid for Optoelectronics Applications. American Journal of Optics and Photonics. Vol. 2, No. 5, 2014, pp. 65-68. doi: 10.11648/j.ajop.20140205.11

\begin{abstract}
In present investigation Hippuric Acid (HA) crystal has been grown by slow evaporation technique. The crystalline nature of the grown crystal was confirmed using single X-ray diffraction technique. The optical studies were carried to examine optical transparency and determine the optical constants of the grown crystal quenching the optoelectronics applications. The grown crystal was subjected to optical studies. The optical studies ascertained high optical transparency and cut off wavelength was found to be $300 \mathrm{~nm}$ very important for nonlinear applications. The optical band gap of grown crystal is found to be $4.14 \mathrm{eV}$. The optical constants such as extinction coefficient, refractive index, optical band gap, and reflectance were calculated. The observed etch patterns confirm the layer growth mechanism.
\end{abstract}

Keywords: Growth from Solution, Hippuric Acid, Single XRD, Nonlinear Optics, Optical Properties

\section{Introduction}

In recent years growth and studies of the organic NLO materials has been emphasized due to their important physical and chemical properties essential for electro-optic modulation, optical switching, high laser damage threshold, second harmonic generation and NLO applications. Nonlinear optics (NLO) is an innovative area of research and development which will play a key role in the field of optoelectronics and photonics [1]. The apparent development of semiorganic materials, where the organic ligand is ionically bonded with inorganic host refined the search of new materials with high optical nonlinearities which is an important area due to their optical applications such as optical communication, optical computing, optical information processing, optical disk data storage, laser fusion reaction, laser remote sensing, colour display, medical diagnostics, etc [2]. Nonlinear optical (NLO) materials play a major role in nonlinear optics and in particular they have a great impact on information technology and industrial applications. On account of the large flexibility for molecular design and higher nonlinear optical efficiency, there has been much progress in basic research on organic and semi-organic NLO materials [3-6]. In present investigation HA crystal has been synthesized and characterized by Single X-ray diffraction and optical studies.

\section{Experimental Procedure}

The Hippuric Acid (HA) was taken and dissolved in acetone to prepare the saturated solution. The saturated solution of HA was obtained by dissolving the material with continuous stirring of solution using magnetic stirrer to ensure homogeneous solution. The solution was then filtered using filter paper and transferred to a Petri dish. The prepared solution was allowed to evaporate at room temperature. The growth was initiated following the nucleation kinetics due to slow evaporation of the solvent. The quality of the crystal was improved by recrystallization process. After a period of 25 days, good quality of the crystal was harvested with more transparency.

\section{Single Crystal X-Ray Diffraction Analysis}

Single crystal X-ray diffraction (XRD) is a non-destructive tool to analyze crystal structure of compounds, which can be grown as single crystals. XRD is employed for finding unit cell parameters, space groups and three-dimensional coordinates of atoms in the unit cell. The grown HA crystals 
were subjected to single crystal X-ray diffraction studies using Enraf nonius single crystal X-ray diffractometer to determine the cell parameters. From the single crystal X-ray diffraction analysis, the crystal belongs to orthorhombic system and the lattice parameters are $\mathrm{a}=8.05 \AA, \mathrm{b}=9.07 \AA$, c $=10.28 \AA$ and the space group is $\mathrm{P} 22_{1} 2_{1} 2_{1}$.

\section{UV- Visible Studies}

A double beam UV-Visible NIR spectrophotometer consists of deutrium lamp which gives continuous radiation and a tungsten as required. A narrow bandwidth was selected with the help of band pass filters. The instrument has an arrangement for switching on either radiation source was passed through the cells of the given solution and solvent alternatively and respectively. This process is called double beam process and it eliminates the fluctuations of intensity of radiation scattering effect and solvent effect. The two rays were combined and differences in intensities of those two radiations are measured electrically. The movement of monochromators and the recorder were synchronized and adjusted so that the record shows the intensity of appropriate radiation. In the transmission study the intensity was recorded in terms of percentage of transmittance (\%) along Y-axis and the wavelength $(\mathrm{nm})$ along $\mathrm{X}$-axis. In this investigation, PerkinElmer double beam UV-VIS-NIR Spectrometer was used to study the optical quality of the grown crystals in the wavelength range of 200 to $1100 \mathrm{~nm}$ at room temperature.

\section{Optical Studies of HA}

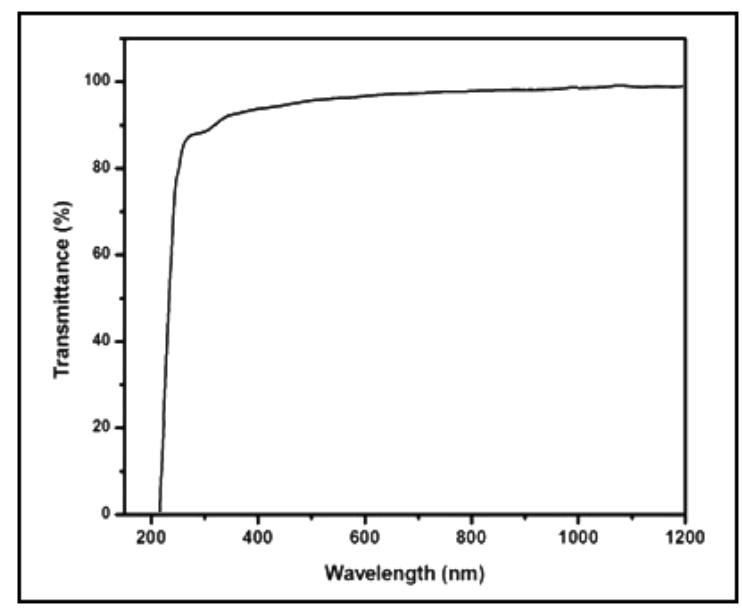

Fig 1. Optical transmission Spectrum of $H A$.

The UV-Visible spectral study of HA crystal was carried out using Perkin-Elmer Lambda 35 spectrophotometer in the range 200-1200 $\mathrm{nm}$ and the recorded transmittance is shown in Fig 1. The grown crystal exhibits high transmittance above $85 \%$ in entire visible region. The lower cutoff wavelength is found to be $300 \mathrm{~nm}$ indicating the wide optical transmission window favorable for second harmonic generation [7]. It shows that the grown crystal has a good transparency in UV, visible and near IR region indicating that it can be used for NLO applications. Hence it is concluded that the grown crystal can be used for optoelectronic applications.

The absorption coefficient is calculated using the transmittance spectrum as

$$
\alpha=\frac{2.303 \log \left(\frac{1}{T}\right)}{d}
$$

where $\mathrm{T}$ is the transmittance, $\alpha$ is the absorption coefficient; $\mathrm{d}$ is the thickness of the crystal optical band gap $\left(\mathrm{E}_{\mathrm{g}}\right)$ depicted in fig. 2 was calculated by

$$
\alpha=A\left(h v-E_{g}\right)^{1 / 2}
$$

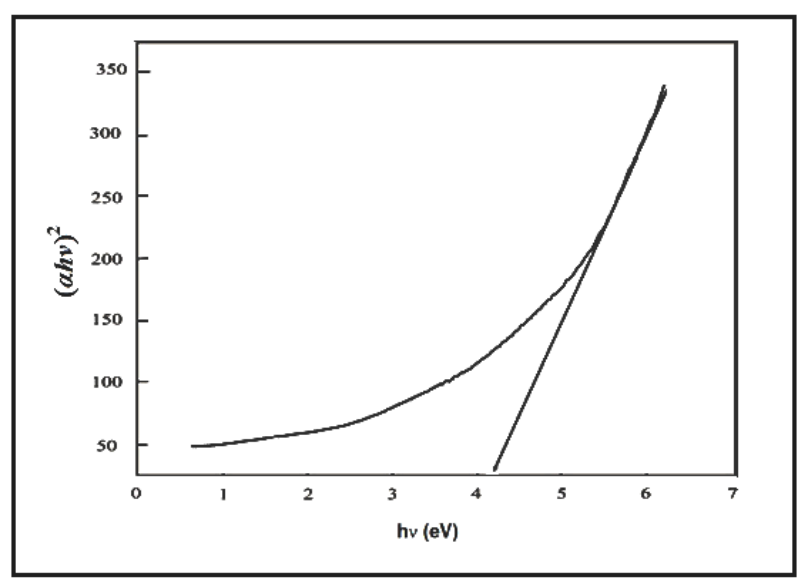

Fig 2. Determination of optical band gap.

The dependence of the optical absorption coefficient $(\alpha)$ on the photon energy ( $h v)$ used to study the band structure and the types of transitions of electrons. The electronic band transitions can be well understood from the plot of $(\alpha h v)^{2}$ verses photon energy as depicted in Fig 2. The wide optical band gap of HA crystal is found to be $4.12 \mathrm{eV}$ suggesting its suitability for optoelectronics applications $[8,9]$.

\subsection{Determination of Optical Constants}

The extinction coefficient $(K)$ can be obtained from the following equation,

$$
K=\frac{\lambda \alpha}{4 \pi}
$$

The transmittance $(T)$ is given by

$$
T=\frac{(1-R)^{2} \exp (-\alpha t)}{1-R^{2} \exp (-2 \alpha t)}
$$

Reflectance $(R)$ in terms of absorption coefficient can be obtained from the above equation. Hence,

$$
R=\frac{1 \pm \sqrt{1-\exp (-\alpha t+\exp (\alpha t)}}{1+\exp (-\alpha t)}
$$


Refractive index $(n)$ can be determined from reflectance data using the following equation,

$$
n=-\frac{(R+1) \pm \sqrt{3 R^{2}+10 R-3}}{2(R-1)}
$$

Reflectance in terms of refractive index (n) is given by relations respectively,

$$
R=\frac{(n-1)^{2}}{(n+1)^{2}}
$$

The refractive index $(n)$ was found to be 1.72 at $\lambda=1200$ $\mathrm{nm}$. From the optical constants, electric susceptibility $\left(\chi_{c}\right)$ can be calculated according to the following relation

$$
\varepsilon_{r}=\varepsilon_{0}+4 \pi \chi_{C}=n^{2}-k^{2}
$$

Hence,

$$
\chi_{C}=\frac{n^{2}-k^{2}-\varepsilon_{0}}{4 \pi}
$$

where $\varepsilon_{0}$ is the permittivity of free space. The value of electric susceptibility $\chi_{C}$ is 0.134 at $\lambda=1200 \mathrm{~nm}$. The real part dielectric constant $\varepsilon_{r}$ and imaginary part dielectric constant $\varepsilon_{i}$ can be calculated from the following relations $\varepsilon_{r}=n^{2}-k^{2}$

$$
\varepsilon_{i}=2 n k
$$

The value of real $\varepsilon_{r}$ and $\varepsilon_{i}$ imaginary dielectric constants at $\lambda=1200 \mathrm{~nm}$ were estimated as 1.263 and $3.403 \times 10^{-5}$, respectively. The moderate values of refractive index and optical band gap suggest that the material has the required transmission range for NLO application. The lower value of dielectric constant and the positive value of the material are capable of producing induced polarization due to intense incident light radiation.

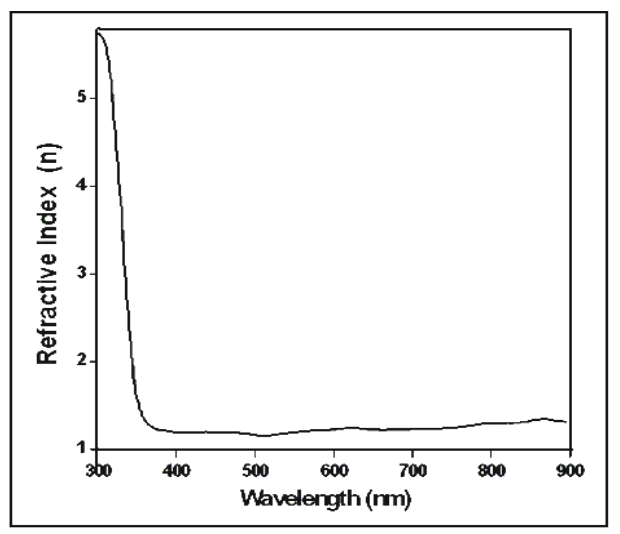

Fig 3. Refractive Index vs. Wavelength.

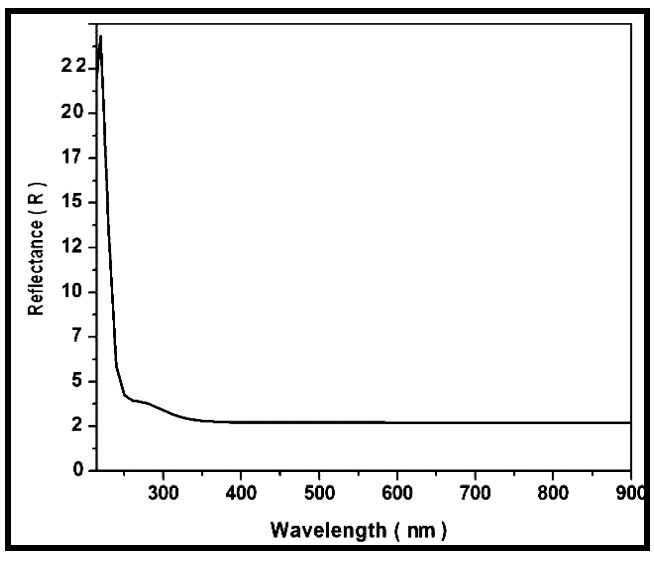

Fig 4. Reflectance vs. Wavelength.

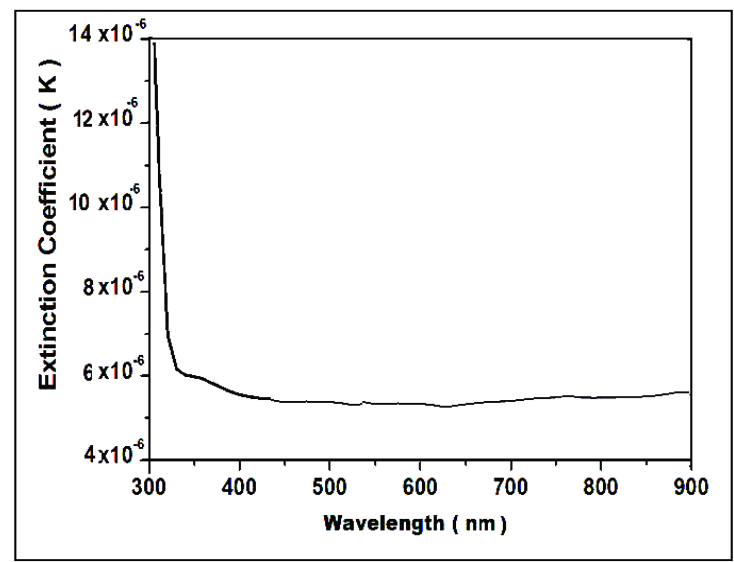

Fig 5. Extinction Coefficient vs. Wavelength.

Figs 3-5 display the dependence of reflectance (R), the extinction coefficient (K) and refractive index (n) on the wave length $(\lambda)$. The high value of refractive index and low value of reflectance reveal that the grown crystal is more transparent to transmit the light from 200 to $1000 \mathrm{~nm}$. Fig. 5 shows the plot of wavelength (nm) versus extinction coefficient $(\mathrm{K})$. It is observed that as the wavelength increases, extinction coefficient decreases up to $200 \mathrm{~nm}$ and remains constant thereafter. This means the absorbance is low in the visible and near IR region. This prediction is also confirmed from UV-VIS-NIR spectrum. Hence the grown crystal HA can easily transmit the wavelength range from 200 to $1200 \mathrm{~nm}$. The large transmittance of the grown crystal is confirmed from the higher values of reflectance and extinction coefficient in the visible region. Moreover, the refractive index of the crystal in the visible region which provides constant transmittance to develop SHG with more efficiency in the device fabrication [10]. Thus HA crystal is prominent material for NLO applications [11].

\section{Etching Studies}

The study of the identification, origin and characteristic of crystalline defects such as boundaries, slip planes, dislocation and plastic flow relies heavily on etching phenomena. Etching is the selective dissolution of the crystal, 
a reverse phenomenon of growth. When a crystal phase is exposed to a solvent, dissolution begins by the nucleation of unit pits of one molecular depth which then grows in the size by the retreating steps across the crystal surface. Etching study was performed on HA by dipping the crystal in water for a few seconds at room temperature. Etch patterns were photographed under an optical microscope in the reflected light and the observed well defined parallel etch pits found as shown in Fig. 6. When etched with water for 10 second, shape of the observed etch pits is close to parallel and layer growth. The shape of the etch pattern indicates the direction of the dislocation lines. Further increasing the etching time to 30 s the pattern remains the same but the numbers of etch pits decrease. While increasing the etching time observed etch patterns are of well defined shape.

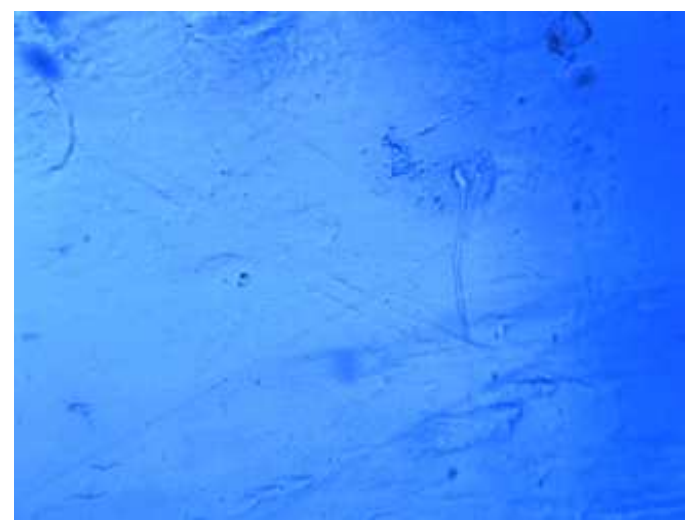

Fig. 6. Etch patterns observed of HA Single Crystal

\section{Conclusion}

The HA crystal was grown by slow evaporation technique. The cut off wavelength was found to be $300 \mathrm{~nm}$. The HA crystal has wide optical band gap of $4.12 \mathrm{eV}$ which makes it suitable for optoelectronic device fabrications. The lower refractive index, extinction coefficient, confirms good optical quality and low defect concentration of the grown crystal. All above studies revealed that the grown crystal is suitable for optoelectronics device fabrication, second harmonic generation and NLO applications. Etching studies on HA reveals very less defects on the surface of the crystal.

\section{Acknowledgement}

The author thanks the Management and Principal of Sree Sastha Institute of Engineering and Technology, Chembarambakkam, Chennai -600123 for their encouragements throughout the work.

\section{References}

[1] Manoj, K.G., Nidhi, S.,and Binay, K.,Growth and characterization of new semi-organic L-proline strontium chloride monohydrate single crystals.Physica B., 2011,406 (1), 63-67.

[2] Maadeswaran, P., Chandrasekaran, J.,and Thirumalairajan,S., Synthesis, growth, spectral, thermal and photoluminescence properties of a new semiorganic NLO crystalThiosemicarbazide lithium chloride [TSLC].Optik.,2011, 122 (3),259-262.

[3] Bright, K.C.,and Freeda,T.H.,Growth and characterization of organometallic L-alanine cadmium chloride single crystal by slow evaporation technique.Physica B., 2010, 405 (18),38573861 .

[4] Balasubramanian, D., Murugakoothan, P., and Jayavel, R., Synthesis, growth and characterization of organic nonlinear optical bisglycine maleate (BGM) single crystals.J. Cryst.Growth. 2010, 312 (11), 1855-1859.

[5] Moitra, S., and Kar,T., Growth and characterization of Lvaline- a nonlinear optical crystal.Cryst. Res. Technol., 2010,45(1),70-74.

[6] Sagadevan S (2014) Growth, optical, mechanical and electrical studies of nonlinear optical single crystal: potassium para-nitrophenolate dihydrate. Science Postprint 1(1): e00026. doi: $10.14340 /$ spp.2014.07A000

[7] Suresh S (2014) Growth, optical and electrical studies of the nonlinear optical crystal: Glycine thiourea. Optik International Journal for Light and Electron Optics 125 (3), pp.950-953.

[8] Suresh S (2014) Studies on the optical and dielectric properties of a zinc thiourea chloride NLO single crystal. Optik - International Journal for Light and Electron Optics 125 (3): pp. 1223-1226

[9] Suresh S (2014) Growth, optical, mechanical, dielectric and theoretical properties of picolinium maleate NLO single crystal. Optik - International Journal for Light and Electron Optics 125 (12): pp. 2826-2829.

[10] S. Suresh and D. Arivuoli, Synthesis, Optical and Dielectric Properties of Tris-Glycine Zinc Chloride Single Crystals, Journal of Minerals \& Materials Characterization \& Engineering, Vol. 10, No.6, pp.517-526, 2011 (TGZC)

[11] Sagadevan, S. and Murugasen, P. (2014) Studies on Optical, Mechanical and Electrical Properties of Organic Nonlinear Optical p-Toluidine p-Toluenesulfonate Single Crystal. Journal of Crystallization Process and Technology,4, 99-110. 